\title{
Assessment of repeated microarray experiments using mixed tissue RNA reference samples
}

\author{
M. Ann Mongan'1, Marnie A. Higgins², P. Scott Pine ${ }^{3}$, Cynthia A. Afshari², and \\ Hisham K. Hamadeh²
}

BioTechniques 45:283-292 (September 2008)

doi $10.2144 / 000112914$

\begin{abstract}
Genome-scale gene expression technologies are increasingly being applied for biological research as a whole and toxicological screening in particular. In order to monitor data quality and process drift, we adopted the use of two rat-tissue mixtures (brain, liver, kidney, and testis) previously introduced as RNA reference samples. These samples were processed every time a microarray experiment was hybridized, thereby verifying the comparability of the resulting expression data for cross-study comparison. This study presents the analysis of 21 technical replicates of these two mixed-tissue samples using Affymetrix RAE230_2 GeneChip over a period of 12 months. The results show that detection sensitivity, measured by the number of present and absent sequences, is robust, and data correlation, indicated by scatter plots, varies little over time. Receiver operating characteristic (ROC) curves show the sensitivity and specificity of the current measurements are consistent with arrays previously classified as well performing. Overall, this paper shows that the inclusion of standard samples during microarray labeling and hybridization experiments is useful to benchmark the performance of microarray experiments over time and allows discovery of any process drift that, if it occurs, may confound the comparison of these datasets.
\end{abstract}

\section{INTRODUCTION}

Microarray analyses have become an integral part of biological studies and, in particular, toxicogenomics. In many contexts, libraries of samples and expression data are generated as references for classifying a sample in question (1-3). Within the drug discovery and investigative toxicology context, such libraries are used to help classify and predict potential toxicities of a compound based on expression data of known toxicants. Success of this approach is dependent on the reliability and reproducibility of sample preparation and analysis within the chosen array technology. However, there have been ongoing concerns about the comparability of microarray data across studies even when the same platform is deployed $(4,5)$. The value of and confidence in genomic data will be greatly increased if there are reliable comparison and integration of results across experiments. In particular, the MicroArray Quality Control (MAQC) project $(6,7)$ was initiated to provide operational metrics for assessing data quality, and standards such as Minimum Information About a
Microarray Experiment (MIAME) $(8,9)$ have been recommended to facilitate the interpretation and reproduction of microarray data. While many of these studies have examined the concordance of microarray data generated across different platforms and laboratories (10-12), much less attention has been paid to the comparability of data generated from the same platform and laboratory over time. Assurance of data comparability in the latter case could be achieved through the use of standardized RNA materials that serve as references for data precision and accuracy.

Previously, we collaborated with the Food and Drug Administration (FDA) and a number of other organizations to introduce two mixed-tissue RNA reference samples, each composed of different amounts of rat brain, liver, kidney, and testis (13). In particular, the ratios of individual tissues between Mix 1 and Mix 2 were brain 2:1, liver 1.5:1, kidney $1: 1$, and testis $1: 4$. If gene transcripts predominantly expressed in only one tissue of the mixtures produced signals proportional to the relative amount of that tissue, the intensity ratio of such a tissue-selective

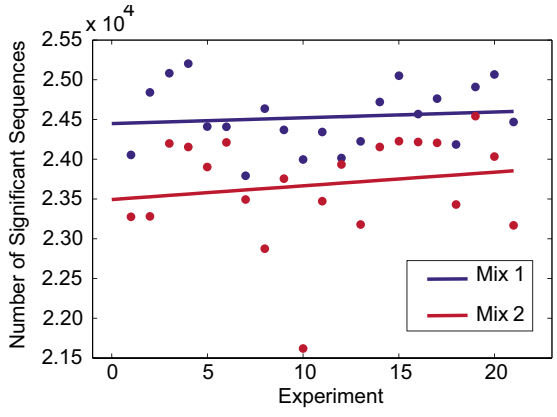

Figure 1. Number of significant sequences in Mixes 1 and 2. Significant sequences are defined as those with statistically significant intensity, $P$ value $\leq 0.1$, based on the Rosetta Resolver error model. The slopes and the $95 \%$ confidence intervals of the two regression lines are $7 \pm 31$ (Mix 1, blue) and $17 \pm 50$ (Mix 2, red).

sequence between Mixes 1 and 2 in a particular experiment would indicate the accuracy of that microarray experiment. Accordingly, the ratios across experiments would indicate the precision of the technology and our laboratory practice over time. Single-use aliquots of each mix were prepared from the same four rat tissues and frozen. One sample of Mix 1 and one of Mix 2 were co-processed with each microarray experiment to monitor array performance as a function of time, operation, protocol, and reagent.

${ }^{1}$ Department of Comparative Biology and Safety Sciences, Amgen, Inc., South San Francisco, ${ }^{2}$ Thousand Oaks, CA, and ${ }^{3}$ Center for

Drug Evaluation and Research, United States Food and Drug Administration, Silver Spring, MD, USA 


\section{Research Reports}
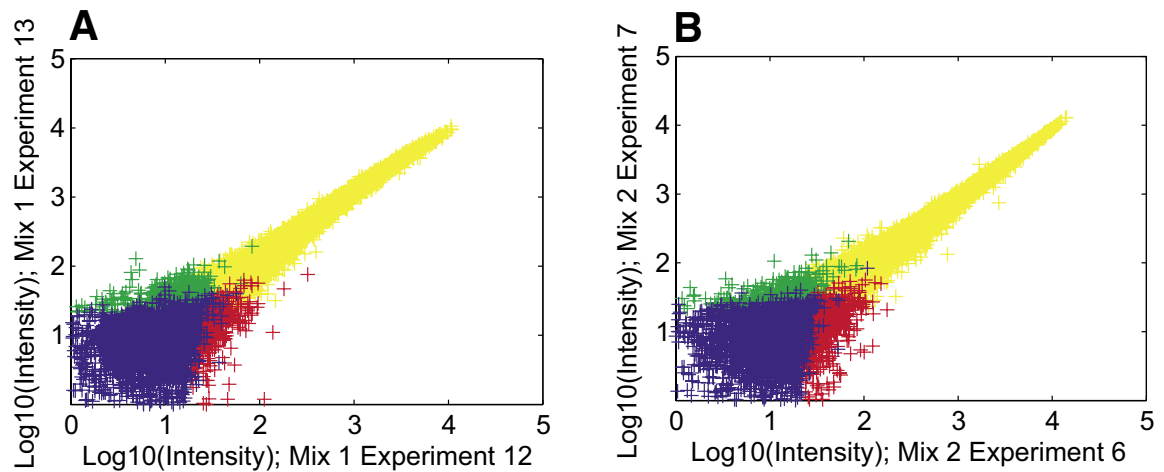

Figure 2. Representative scatter plots of Mix 1 and Mix 2 arrays. (A) Two arrays of Mix 1. (B) Two arrays of Mix 2. Sequences with $P$ value $\leq 0.1$ were determined as present. Yellow markers identify sequences present in samples on both $\mathrm{x}$ - and $\mathrm{y}$-axes; green for sequences present only in sample on the $y$-axis; red for sequences present only in sample on the $x$-axis; and blue for sequences absent in both samples.

\section{A}

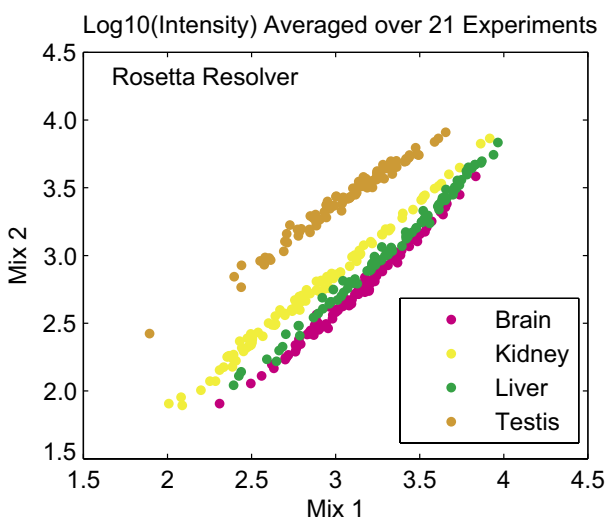

\section{B}

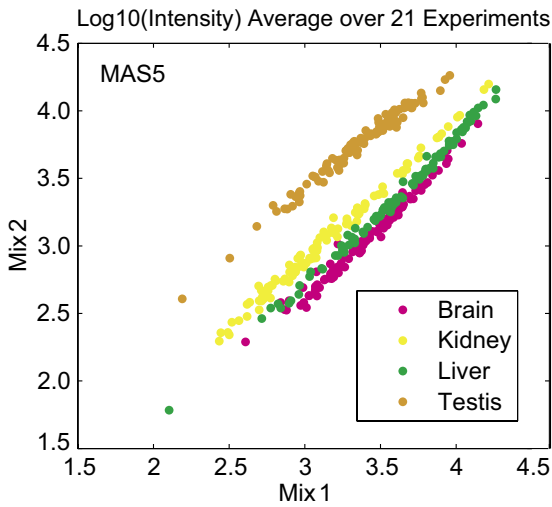

Figure 3. Scatter plots of tissue-selective sequences between Mixes 1 and 2. Signal intensities from brain-selective (pink), kidney-selective (yellow), liver-selective (green), and testis-selective (orange) were averaged across the 21 arrays. (A) Data processed with the Rosetta Resolver's error model. (B) Data processed with MAS5. Intensities were $\log _{10}$-transformed for easy comparison with published results (13). The pre-processing method shown in (A) was used for all analyses in this study.

All samples discussed in this study were run on Affymetrix RAE230_2 (Affymetrix, Santa Clara, CA, USA) at a single microarray laboratory. This paper presents the results of the analysis, based on previously described metrics (14), of gene expression obtained from these reference samples over a 12-month period.

\section{MATERIALS AND METHODS}

\section{Sample Preparation and RNA Isolation}

Tissues (liver, kidney, brain, and testis) were harvested from eight naïve male Sprague-Dawley rats and diced before being submerged in RNAlater stabilization solution (Ambion, Austin, TX, USA). Preserved tissue samples were handled according to the manufacturer's instructions and stored at $-80^{\circ} \mathrm{C}$ until processed. Total RNA was isolated according to the RNeasy extraction procedure (Qiagen, Valencia, CA, USA). Briefly, tissues were homogenized in RLT lysis buffer containing $\beta$-mercaptoethanol using a handheld rotor-stator homogenizer. Sample homogenates were applied to RNeasy Maxi columns (Qiagen) and processed according to the manufacturer's instructions. An on-column DNase digestion was performed to remove any residual genomic DNA contamination. RNA samples were eluted in RNase-free water, and concentration was measured spectrophotometrically using the Biophotometer (Eppendorf, Westbury, NY, USA). Nucleic acid quality was evaluated with the RNA 6000 Nano Chip kit (Expert software; Agilent Technologies, Santa
Clara, CA, USA). After confirming the quality of RNA samples from individual rats, a pooled sample containing equal amounts of RNA from each of the eight replicate animals was prepared for each of the four tissue types. Following quality assessment of the pooled samples, two mixed-tissue standards were prepared according to the following specifications: Mix 1 contained $40 \%$ brain, $30 \%$ liver, $20 \%$ kidney, and $10 \%$ testis; Mix 2 contained $20 \%$ brain, $20 \%$ liver, $20 \%$ kidney, and $40 \%$ testis. All samples were of high quality, as evidenced by distinct ribosomal $18 \mathrm{~S}$ and $28 \mathrm{~S}$ peaks, absence of extraneous peaks, and low baseline values on the electropherogram. RNA integrity number (RIN) values were 9.2 and 9.4 for Mix 1 and Mix 2, respectively. To eliminate the potential effect of freeze-thaw cycles on RNA quality, single-use aliquots of the mixed-tissue standards were prepared and stored at $-80^{\circ} \mathrm{C}$ until being processed for microarray hybridization.

\section{Gene Expression Measurement}

For microarray profiling, $5 \mu \mathrm{g}$ of total RNA was reverse-transcribed to double-stranded cDNA, and biotinylated cRNA was generated using the BioArray HighYield RNA Transcript Labeling kit (Enzo Life Sciences, Farmingdale, NY, USA). To increase hybridization efficiency $(\sim 16 \mathrm{~h})$, cRNA samples were fragmented according to standard Affymetrix protocols before being incubated overnight with the GeneChip Rat Genome 230 2.0 Array (Affymetrix). Arrays were washed and stained using the antibody amplification and staining protocol according to the vendor's instructions.

\section{Data Analysis}

Reporter-level data were archived in Rosetta Resolver 6.0 (Rosetta Biosoftware, Seattle, WA, USA). Present and absent sequence calls made by Rosetta Resolver were based on an error model that made use of negative control probes (AFF-xxxx_st) on the chip. Sequence-level intensity data, obtained by Rosetta Resolver's squeezing process, were imported into Matlab 7.4.0 (The Mathworks, Natick, MA, USA) for analysis. Expression 
values were $\log _{2}$-transformed and quantile-normalized across profiles of the same mix. MAS5 normalized data were obtained using the mas 5 function of the Bioconductor affy package in R. MAS5 normalized data were used only to generate Figure 3B. One hundred tissue-selective sequences were identified for each of the four tissues: brain, liver, kidney, and testis (14). $\log _{2}$ ratios of Mix 1 and Mix 2 were further normalized by the $10 \%$ trimmed mean of kidney-selective sequences. The number of present sequences as a function of time was calculated using linear regression in Matlab. Scatter plots between consecutive profiles were generated using the scatter plot function in Rosetta Resolver. Receiver operating characteristic (ROC) curves plotted sensitivity as a function of (1-specificity). Sensitivity and specificity were defined by Equations 1 and 2, respectively.

sensitivity $=\frac{\text { true positive }}{\text { true positive }+ \text { false negative }}$

[Eq. 1]

specificity $=\frac{\text { true negative }}{\text { true negative }+ \text { false positive }}$

[Eq. 2]

By definition, all of the liver-, brain-, and testis-selective sequences are true positives because they represent 1.5-, $2-$, and 4-fold designed-in changes; and all of the kidney-selective sequences are true negatives because they are designed to be $1: 1$ between the mixtures. The ROC curve was generated by averaging the $\log _{2}$ ratio of each sequence over 21 experiments (Equation 3) and varying the cutoff value between -1 and 2.5 with a 0.1 increment. The cutoffs were used to determine the fraction of true positives and true negatives detected by the assay.

mean $\log _{2}$ ratio of sequence $i=$

$$
\frac{1}{N} \sum_{j=1}^{N} \log _{2} I_{i, j}^{M i x 1}-\log _{2} I_{i, j}^{M i x 2}
$$

[Eq. 3] where $I_{i, j}$ is the intensity of sequence $i$ in experiment $j$ and $n=21$ is the total number of experiments in our analysis. In the ROC plot, the ratios of Mix 2/Mix 1 for testis-specific sequences were calculated instead because the testis tissue was present in a higher percentage in Mix 2 . Optimal performance of the arrays would identify all true positives before any false positives, yielding an ROC plot with an area under the curve (AUC) equal to 1 . The process drift plot monitored the area under the ROC curve as a function of time. The box and whisker plot was generated by comparing the mean intensity ratios of tissue-selective sequences to the relative amount of the tissues in the mixes. Each box on this plot showed lines at the lower quartile, median, and upper quartile values. The whisker lines extended from each end of the boxes to the most extreme values within $1.5 \times$ of the interquartile range. Values beyond the ends of the whiskers were considered outliers.

\section{RESULTS}

\section{Array Quality}

A total of 42 arrays were included in this study. The 21 arrays of each mix were technical replicates processed at different time points. The hybridized chips were scanned and .cel files were imported into the Rosetta Resolver system. The .cel files were also submitted to the Gene Expression Omnibus (GEO) database (Accession no. GSE11542). The number of good reporters, determined by the Affymetrix scanner, on each array was about $99.9 \%$ of the 342,410 total reporters on the chip. A reporter is equivalent to an Affymetrix probe pair. Present reporters and sequences were those determined significant by the Rosetta Resolver's error model at the $P$ value threshold of 0.1 . Figure 1 shows the number of significant sequences in arrays collected over the 12-month period. The 95\% confidence interval of the slopes of regression lines include zero, indicating that no positive or negative trends were significant with the current dataset. The $\mathrm{R}^{2}$ of the trend lines are 0.01 and 0.03 for Mixes 1 and 2, respectively, suggesting that the detection sensitivity is stable and precise. This result indicates the

\section{BioTechniques}

Connecting. Informing. Advancing. For 25 Years.

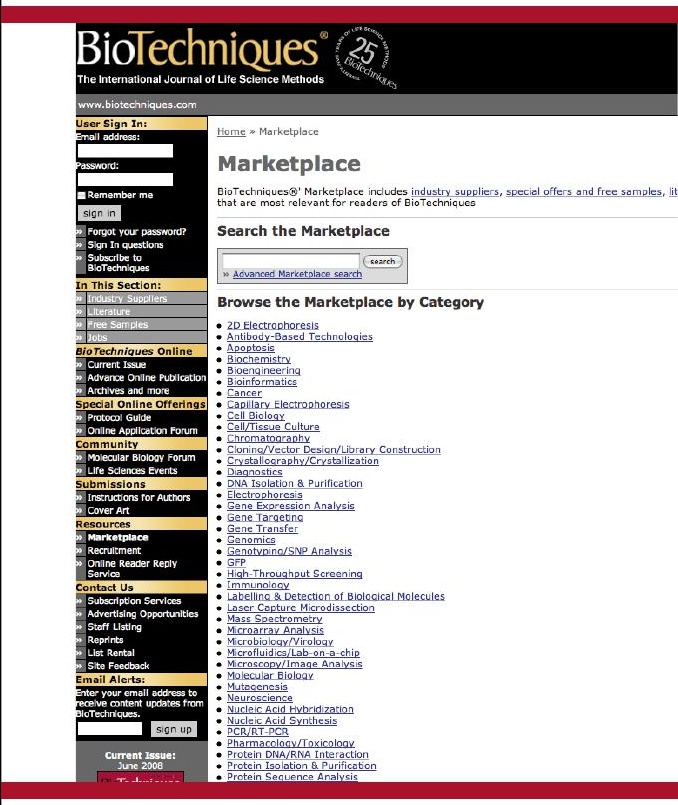

\section{Online Marketplace}

- Search industry suppliers

- Request commercial literature

- Browse special offers and free samples

Check out the BioTechniques Online Marketplace at www.biotechniques.com/marketplace 


\section{BioTechnipues}

Connecting. Informing. Advancing.

For 25 Years.

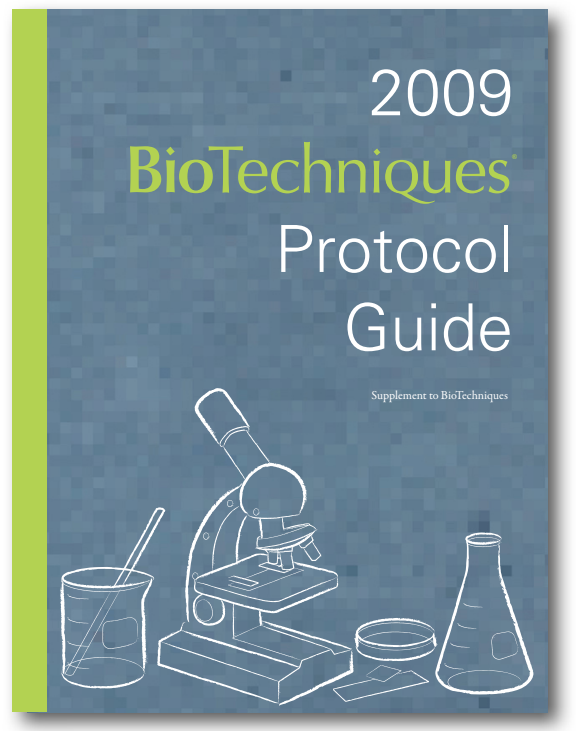

A

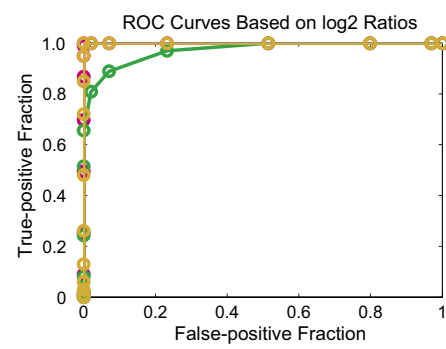

B

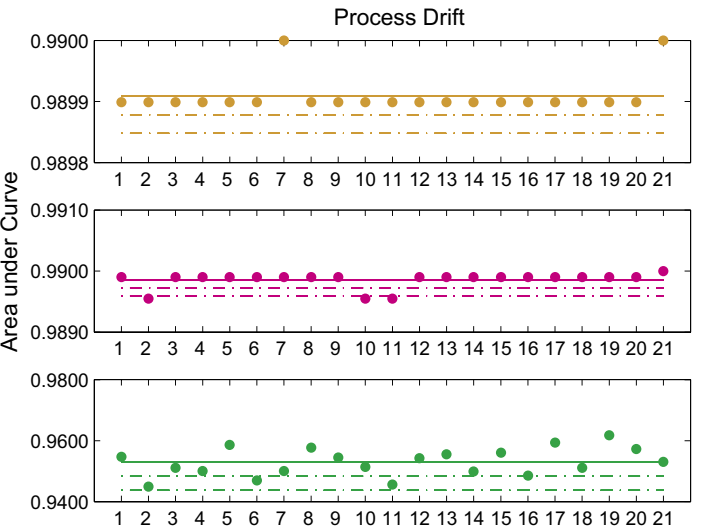

Figure 4. Sensitivity and selectivity of RAE230_2 arrays as measured by tissue-selective sequences. (A) Receiver operating characteristic (ROC) curves. ROC curves were generated by considering the number of sequences correctly identified as true positive or true negative relative to false positive and negative in each array and then averaging over the 21 arrays for each tissue; brain (pink), liver (green), and testis (orange). (B) Process drift as measured by ROC plots. Each data point corresponds to the area under the curve (AUC) of the ROC curve generated by tissue-selective sequences in each of the 21 experiments. Higher AUC values reflect higher sensitivity and selectivity. Due to the higher tissue ratios between the two mixes, brain (pink) and testis (orange) have AUC values closer to 1 than liver (green) does. The solid line indicates the AUC mean in each plot; the two dotted lines indicate one and two standard deviations from the mean.

good precision of our array processing, which is expected because the mixes were derived from the same biological samples. The background intensities were estimated by Rosetta Resolver based on the lowest $2 \%$ reporter intensities. The mean background intensities were relatively constant across the 21 arrays of each mix and were about $1 \%$ of the mean intensities.

Before normalization, scatter plots were generated to assess the correlation between chronologically consecutive arrays derived from the same mix. These plots showed that arrays of the same mix were highly and consistently correlated (Figure 2, A and B). The level of correlation was comparable among samples of Mix $1(r=0.996)$ and those of Mix 2 $(r=0.997)$. This finding was in contrast with the lower correlation $(r=0.749)$ observed when the intensity profile from a sample of Mix 1 was plotted against a sample derived exclusively of liver tissue. Interestingly, the correlation between samples of the same mixes for sequences with low expression level $\left(\log _{10}\right.$ intensity less than 2 ) was also satisfactorily observed with $r>0.7$.

\section{Performance of Tissue-selective Sequences}

When the two rat mixed-tissue samples were first introduced as reference materials, 203 sequences were identified as being tissue-selective on the Affymetrix platform, with $\sim 50$ sequences chosen for each tissue (13). The number of tissue-selective sequences was subsequently expanded to 100 for each tissue (see Supplementary Table S1 available online at www.BioTechniques.com) (14). Figure 3 shows the mean intensity of sequences in Mix 1 against those in Mix 2. The dynamic range of intensities measured in our study (using Affymetrix RAE230_2) was similar to that measured with Affymetrix RAE230A (Figure 3), but was markedly different from those measured with the CodeLink or Agilent platforms, which had $\log _{10}$ intensity ranges of $-2-2$ and $2-5$, respectively (13).

Sensitivity and specificity of our arrays and process, shown by the ROC plot (Figure 4A), are consistent with arrays previously classified as well performing (13). At a particular detection cutoff, all true positives ideally should be identified before any false positives, yielding an ROC curve that consists of a vertical line at $\mathrm{x}=0$ and a horizontal line at $y=1$. Accordingly, the AUC of this ideal ROC curve is 1 , while that resulting from a random process is 0.5 . The AUC calculated for the ROC curves of testis, brain, and liver were $0.99,0.99$, and 0.96 , respectively. This finding indicates that the current technology can detect a 


\section{Research Reports}

A

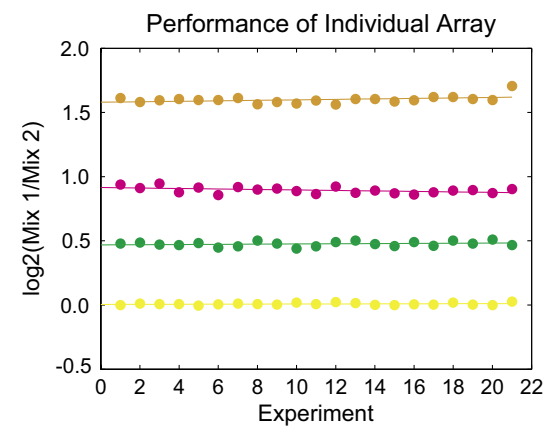

B

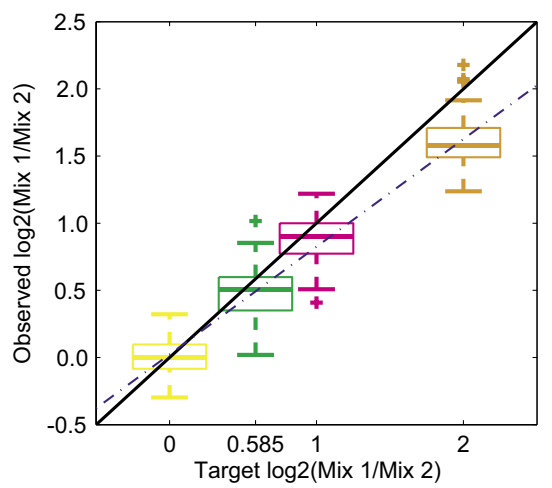

Figure 5. $\log _{2}$ ratios of tissue-selective sequences as compared with relative ratio of the four tissues between Mixes 1 and 2. (A) Each data point is calculated by taking the ratio $\log _{2}$ (Mix 1/Mix 2 ) averaging over the 100 sequences of the same tissue in each experiment. Best-fitted lines were drawn for each tissue. (B) Each box and associated whiskers span the range of 100 data points, each represents the $\log _{2}$ intensity ratio of the same sequence averaged over 21 technical replicates. Lines in each box represent the lower quartile, median, and upper quartile values of the corresponding range. The dotted line is the best fitted line for all data points while the solid line marks $\mathrm{y}=\mathrm{x}$.

difference of 2-fold (brain) in sequence abundance as well as 4-fold (testis). The process drift plots (Figure 4B) monitor the performance of each tissue over the 12-month period. Brain- and testisspecific sequences were consistently measured with good accuracy, with AUC at 0.99 for most experiments. Signals for liver-specific sequences, though having smaller ratio difference between the two mixes, were also well detected with all AUC greater than 0.94.

To further assess process drift, we calculated the mean $\log _{2}$ ratio of each tissue-selective sequence group in the same experiment (Figure 5A). The resulting ratios were consistent across all experiments with the best-fitted lines having slopes within \pm 0.003 . Since Mix 1 and 2 were normalized with the $10 \%$ trimmed mean of kidney-selective sequences, the ratios of these sequences were close to unity for all 21 arrays. However, the measured intensity ratios of liver, brain, and testis were all lower than the relative amount of tissues in the two mixes (the target ratios), suggesting that measured intensities did not scale perfectly with tissue abundance. The box and whisker plot (Figure 5B) shows the $\log _{2}$ ratio of each tissue, across repeated experiments, as compared with the target ratios between the two mixes. The lower slope of the best-fitted line relative to the $\mathrm{y}=\mathrm{x}$ line again indicated a non-perfect correspondence between measured intensity and tissue ratio, a compression effect similar to what had been documented for microarray as well as a number of PCR platforms (13). We explore possible effects contributing to this observation in our discussion section.

\section{DISCUSSION}

This paper describes the analysis of a quality control process we developed for microarray data. Though Affymetrix affirms that its GeneChip technology is reliable and robust (15), our process allows us to monitor not only the technology but also the quality of our microarray laboratory over time. The samples analyzed in this paper were two rat-tissue mixtures with known relative proportions of brain, liver, kidney, and testis RNA. One sample of each tissue mixture was processed every time we carried out a gene expression experiment, which was approximately 2 weeks apart.

We found that the RAE230_2 GeneChip delivers a similar dynamic range as compared to the previous rat array (RAE 230). The average intensities of tissue-selective sequences over repeated experiments indicated their reliability as tissue markers. The large ratio difference between testis- and kidney-selective sequences translated to the distinct separation between these two groups of sequences on this plot. The ratios between brain- and liver-selective sequences were also satisfactorily distinguishable even though the signal difference between these two tissues was much smaller. The effect of the pre-processing algorithm seemed to be minimal, as evidenced by the similarity of intensity distributions created by applying the Rosetta Resolver error model followed by quantile normalization in Matlab (Figure 3A), and those produced by MAS5 (Figure 3B). The main difference between our method and results produced by MAS5 was that the former has a smaller dynamic range. The average signal of the current data, normalized by MAS5, is consistent with the previously published data using the MAS5 and PLIER algorithms (13).

Our results show that Affymetrix arrays are consistent and performed well in terms of detection sensitivity and specificity, as reflected by the ROC plots. The AUC of the ROC plot is a composite measure of the microarray experiment quality, and therefore can be used to identify a potential problematic experiment that needs to be reproduced. The decision to repeat an experiment is, in addition, a trade-off between the cost of repeating experiments that may have been valid and tolerance of error in the results; no single guideline is likely to be ideal for all investigations. Currently, we consider a cutoff of AUC $=0.9$, not as a threshold for automatic repetition of the experiment but as a guideline for further investigation of data quality, because this AUC values strikes a good balance in this trade-off. However, a statistically justified cutoff for declaring an experiment to be repeated is dependent on the probability distribution of sufficiently large AUC values. We are continuing to use these mixed tissues to collect data for such a distribution and plan to conduct more rigorous analysis to determine such a cutoff.

We noticed a compression effect (Figure 5, A and B) that was reproducible in 21 experiments. One explanation for the observed discrepancy between the intensity ratios and the target ratios is that the 400 chosen sequences are not entirely tissue specific and the observed lower ratios reflect contributions from multiple tissues. In addition, limitations at each end of the dynamic range of the scanner could also contribute to ratio compression (16). In silico modeling- 


\section{Research Reports}

derived expected ratios based on pooled tissue data predicted a comparable degree of ratio compression (13). A similar compression effect was previously observed by Canales et al. (16) when these authors compared fold-change correlation between various microarray platforms (including Affymetrix) with TaqMan, StaRT-PCR, and QuantiGene. While these sources of compression are intrinsic to the assay, ratios much less than predicted could also indicate procedural problems that may be correctable (17). The use of these reference RNAs as concurrent controls help us to determine the extent of ratio compression, which is particularly important for interpreting data based on fold changes.

In summary, the present study assesses the performance of Affymetrix RAE230_2, as processed by our laboratory, over a 12-month period, and the accuracy of these experiments based on known ratios of two reference rat-tissue mixtures. Overall, we found good precision and no significant process drift. Analysis of the 400 tissue-selective sequences showed that results collected in our laboratory were equivalent to published arrays considered to have good performance. The intensity ratios were found to be generally lower than the tissue ratio between two mixes, which is probably a result of the low specificity of the chosen sequences or a nonlinear relationship between measured intensity and RNA abundance. The result of this study assures comparability of our microarray data and allows cross-comparison of data from multiple studies. Data in this study represent expected typical results and thus can

be used to benchmark laboratory proficiency. We are continuing to use these reference RNAs to monitor our process and encourage the use of such a quality control in other laboratories.

\section{ACKNOWLEDGEMENTS}

We thank Mike Boedigheimer and Karol L. Thompson for useful comments on data analysis; Annie Kwok for her contribution to the RNA extraction work; Mike Damore, Mara Campbell, Pani Kiaei, Dan Baker, and Chetan Deshpande for generating the microarray data; and Tiffany Day for help with Gene Expression Omnibus (GEO) submission.

\section{COMPETING INTERESTS STATEMENT}

The authors declare no competing interests.

\section{REFERENCES}

1. Barrett, T., D.B. Troup, S.E. Wilhite, P. Ledoux, D. Rudnev, C. Evangelista, I.F. Kim, A. Soboleva, et al. 2007. NCBI GEO: mining tens of millions of expression profilesdatabase and tools update. Nucleic Acids Res 35:D760-D765.

2. Fostel, J., D. Choi, C. Zwickl, N. Morrison, A. Rashid, A. Hasan, W. Bao, A. Richard, et al. 2005. Chemical effects in biological systems - data dictionary (CEBS-DD): a compendium of terms for the capture and integration of biological study design description, conventional phenotypes, and 'omics data. Toxicol. Sci. 88:585-601.

FLUORESCENCE ILLUMinATION

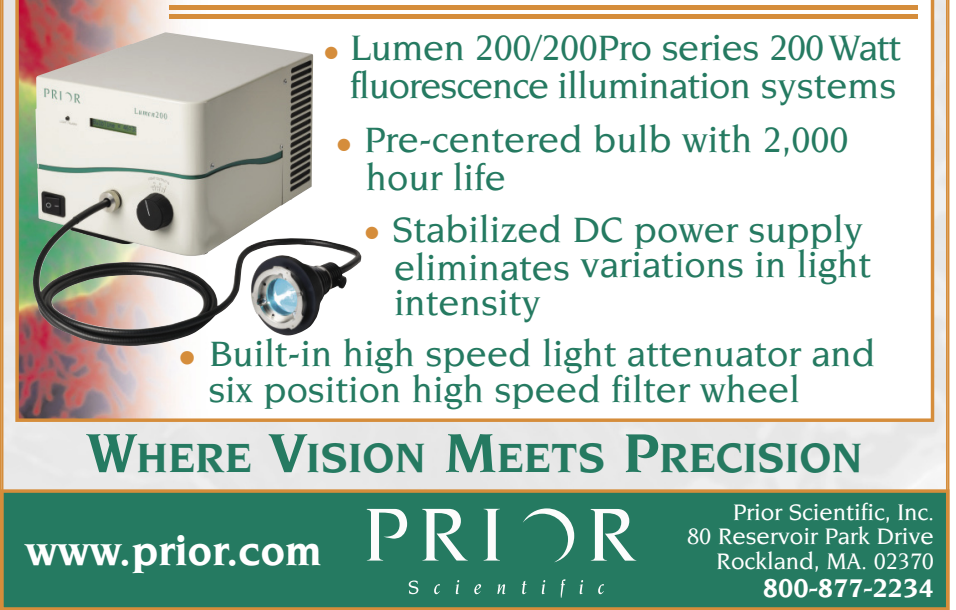

3. Waters, M., S. Stasiewicz, B.A. Merrick, K. Tomer, P. Bushel, R. Paules, N. Stegman, G. Nehls, et al. 2008. CEBS-Chemical Effects in Biological Systems: a public data repository integrating study design and toxicity data with microarray and proteomics data. Nucleic Acids Res. 36:D892-D900.

4. Draghici, S., P. Khatri, A.C. Eklund, and Z. Szallasi. 2006. Reliability and reproducibility issues in DNA microarray measurements. Trends Genet. 22:101-109.

5. Nimgaonkar, A., D. Sanoudou, A.J. Butte, J.N. Haslett, L.M. Kunkel, A.H. Beggs, and I.S. Kohane. 2003. Reproducibility of gene expression across generations of Affymetrix microarrays. BMC Bioinformatics 4:27.

6. Chen, J.J., H.-M. Hsueh, R. Delongchamp, C.-J. Lin, and C.-A. Tsai. 2007 Reproducibility of microarray data: a further analysis of microarray quality control (MAQC) data. BMC Bioinformatics 8:412 


\section{Research Reports}

7. Shi, L., L.H. Reid, W.D. Jones, R. Shippy, J.A. Warrington, S.C. Baker, P.J. Collins, F. de Longueville, et al. 2006. The MicroArray Quality Control (MAQC) project shows inter- and intraplatform reproducibility of gene expression measurements. Nat. Biotechnol. 24:1151-1161.

8. Brazma, A., P. Hingamp, J. Quackenbush, G. Sherlock, P. Spellman, C. Stoeckert, J. Aach, W. Ansorge, et al. 2001. Minimum information about a microarray experiment (MIAME) - toward standards for microarray data. Nat. Genet. 29:365-371.

9. Knudsen, T.B. and G.P. Daston. 2005. MIAME guidelines. Reprod. Toxicol. 19:263.

10. Kuo, W.P., T.K. Jenssen, A.J. Butte, L. OhnoMachado, and I.S. Kohane. 2002. Analysis of matched mRNA measurements from two different microarray technologies. Bioinformatics 18:405-412

11. Bammler, T., R.P. Beyer, S. Bhattacharya, G.A. Boorman, A. Boyles, B.U. Bradford, R.E. Bumgarner, P.R. Bushel, et al. 2005. Standardizing global gene expression analysis between laboratories and across platforms. Nat. Methods 2:351-356.

12. Yauk, C.L., M.L. Berndt, A. Williams, and G.R. Douglas. 2004. Comprehensive com- parison of six microarray technologies. Nucleic Acids Res. 32:e124.

13. Thompson, K.L., B.A. Rosenzweig, P.S. Pine, J. Retief, Y. Turpaz, C.A. Afshari, H.K. Hamadeh, M.A. Damore, et al. 2005. Use of a mixed tissue RNA design for performance assessments on multiple microarray formats. Nucleic Acids Res. 33:e187.

14. Pine, P.S., M. Boedigheimer, B.A. Rosenzweig, Y. Turpaz, Y.D. He, G. Delenstarr, R. Shippy, B. Ganter, et al. 2007. Metrics for assessing the performance of microarray assays using a mixed tissue RNA reference material. Poster for the 46th Annual Meeting of the Society of Toxicology, Charlotte, NC

15. Dalma-Weiszhausz, D.D., J. Warrington, E.Y. Tanimoto, and C.G. Miyada. 2006. The Affymetrix GeneChip platform: an overview. Methods Enzymol. 410:3-28.

16. Canales, R.D., Y. Luo, J.C. Willey, B. Austermiller, C.C. Barbacioru, C. Boysen, K. Hunkapiller, R.V. Jensen, et al. 2006. Evaluation of DNA microarray results with quantitative gene expression platforms. Nat. Biotechnol. 24:1115-1122.

17. Han, T., C.D. Melvin, L. Shi, W.S. Branham, C.L. Moland, P.S. Pine, K.L. Thompson, and J.C. Fuscoe. 2006. Improvement in the reproducibility and accuracy of DNA microarray quantification by optimizing hybridization conditions. BMC Bioinformatics 7(Suppl 2):S17.

Received 20 February 2008; accepted 15 June 2008.

Address correspondence to Ann Mongan, Department of Comparative Biology and Safety Sciences, Amgen, Inc., 1120 Veterans Blvd., Mail stop ASF1-2, South San Francisco, CA 94080, USA. e-mail:thuyv@amgen.com

To purchase reprints of this article, contact: Reprints@BioTechniques.com

MHN OUR ENVRONMENT

THE SAFEST AND SIMPLEST WAY TO

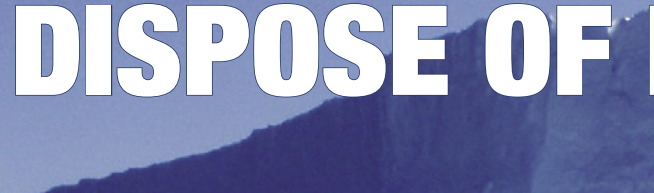

Easy handling - closed system removes $100 \%$ EtBr from aqueous solutions.

High capacity - one cartridge removes

$2 \mathrm{~g}$ of $\mathrm{EtBr}$ and purifies 4000 liters of

EtBr solution.

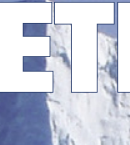

D $\left\{\begin{array}{l}D \\ D\end{array}, \sqrt{ } \square\right.$

\section{A}
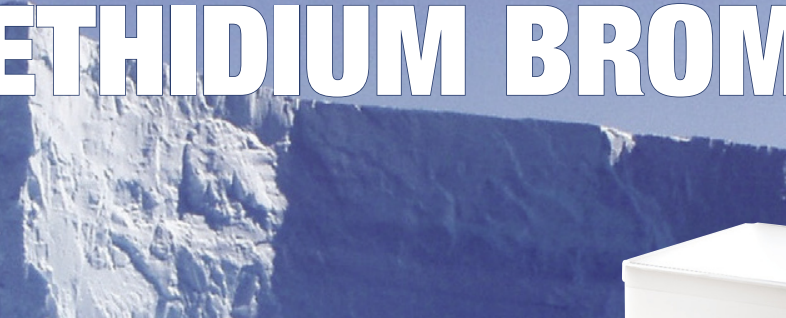